\title{
3D Model Based Pose Invariant Face Recognition from a Single Frontal View
}

\author{
Qinran Chen and Wai-kuen Cham \\ Department of Electronic Engineering, The Chinese University of Hong Kong \\ Shatin, New Territories, Hong Kong
}

Received 2 August 2005 ; accepted 16 March 2007

\begin{abstract}
This paper proposes a 3D model based pose invariant face recognition method that can recognize a face of a large rotation angle from its single nearly frontal view. The proposed method achieves the goal by using an analytic-to-holistic approach and a novel algorithm for estimation of ear points. Firstly, the proposed method achieves facial feature detection, in which an edge map based algorithm is developed to detect the ear points. Based on the detected facial feature points 3D face models are computed and used to achieve pose estimation. Then we reconstruct the facial feature points' locations and synthesize facial feature templates in frontal view using computed face models and estimated poses. Finally, the proposed method achieves face recognition by corresponding template matching and corresponding geometric feature matching. Experimental results show that the proposed face recognition method is robust for pose variations including both seesaw rotations and sidespin rotations.
\end{abstract}

Key Words: Face Recognition, Pose Estimation, 3D face Model, Single View.

\section{Introduction}

During the last few decades, research on the automatic face recognition (AFR) has received increasing attention, and different face recognition algorithms have been developed. [1-3] give some good reviews in this field. Most AFR algorithms are for face recognition under controlled conditions. For example, satisfactory recognition rates on face images which are uncovered, in frontal view, with neutral expression and controlled lighting have been reported in [7-10]. While some other recognition algorithms such as [1315] have been developed to tackle the variations on different lighting, small occlusions, and facial expressions for frontal view face images. The results are encouraging.

The problem related to variations in poses received much attention and many algorithms have been developed to tackle this problem. An early attempt is the 2D appearance based approach which describes faces under varying pose with a set of $2 \mathrm{D}$ features and achieves pose analysis and face recognition by comparing these features. [18] presents a method for pose invariant face recognition in the entire eigenspace. Huang et. al [16] achieved pose invariant face recognition in the view-space which is a subspace of the eigen-space. Demir's method [19] is similar to that of [16], but employing a sub-LDA space as the viewspace. In [11] and [12], this problem was tackled in the discriminant waveletface space and the kernel LDA space respectively. [20] describes a line-based algorithm for pose invariant face recognition. These

Correspondence to: qrchen@ee.cuhk.edu.hk

Recommended for acceptance by E. Martí

ELCVIA ISSN: 1577-5097

Published by Computer Vision Center / Universitat Autonoma de Barcelona, Barcelona, Spain 
appearance based methods can provide good recognition results based on dense sampling of the continuous pose in gallery. However this requirement not only increases the gallery size but also makes the recognition process more time consuming. In addition, when the gallery consists of only one front view image (such as a passport photo) per candidate, these methods cannot work. Therefore the 3D model based approach was proposed. It has stronger generalization to pose variation and is available to achieve pose invariant face recognition from a single frontal view, though its implementation is more complex.

In the 3D model based approach, a 3D face model is built to represent the 3D geometry of human faces in 2D images. This approach removes the effect of pose variations on face recognition by estimating and aligning poses with a 3D face model and then extracting features under a uniform pose for classification. Generally, pose estimation is the most critical and challenging operation in the 3D model based approach. In [21-22], fixed generic 3D face models were proposed to be used for all candidates. These methods can achieve pose estimation from a single face image based on affine transforms. However pose of a particular face cannot be estimated accurately by using a fixed 3D model. In [6, 17], simple adaptive 3D face models that can adapted to fit a particular person were proposed. The pose estimation and the model adaptation were achieved synchronously by using geometrical measurements. These methods can obtain effective pose estimation from a single face image. However, $[6,17]$ can only estimate the sidespin rotations of the face in an image with the assumption that the face has no seesaw rotation. Recently, Blanz et al [23-24] built a 3D morphable face model from a large set of real 3D face data for pose invariant face recognition. Based on this model, the pose estimation and model adaptation were achieved by hybrid geometric information and texture information based optimization. The reported performance of pose estimation in this system is good, but the optimization procedure is very complex and requires large computing time.

This paper proposes a model based pose invariant face recognition method to recognize a face from its single nearly frontal view. As a generalization of Lam and Yan's method [6], our method obtain more robust performance to pose variation and gives following contributions: 1) proposed a edge map based ear point detection algorithm, 2) presents a more general and powerful pose estimation algorithm 3) achieve classification by corresponding template matching and corresponding geometric feature matching.

\section{Overview of the Proposed Method}

In this paper we propose a 3D model based pose invariant face recognition method that can recognize a face from its single nearly frontal view, which assumes that the face has no seesaw rotation and may have small sidespin rotation. The proposed method is composed of four operations: (1) facial feature detection, (2) pose estimation and 3D model adaptation, (3) pose invariant feature extraction, and (4) classification. The block diagram of the proposed method is given in Fig. 1. In the operation of feature detection, beside eye corners, mouth corners, nose tip, eyebrow points, face contour, new facial features in the form of two lower joint points of the ears and the face boundary (called ear points in the following) are detected by an edge map based algorithm. A simple adaptive 3D face model is used to represent the 3D geometry of the face in an image. With the assumption that the gallery faces have no seesaw rotation, we achieve pose estimation and

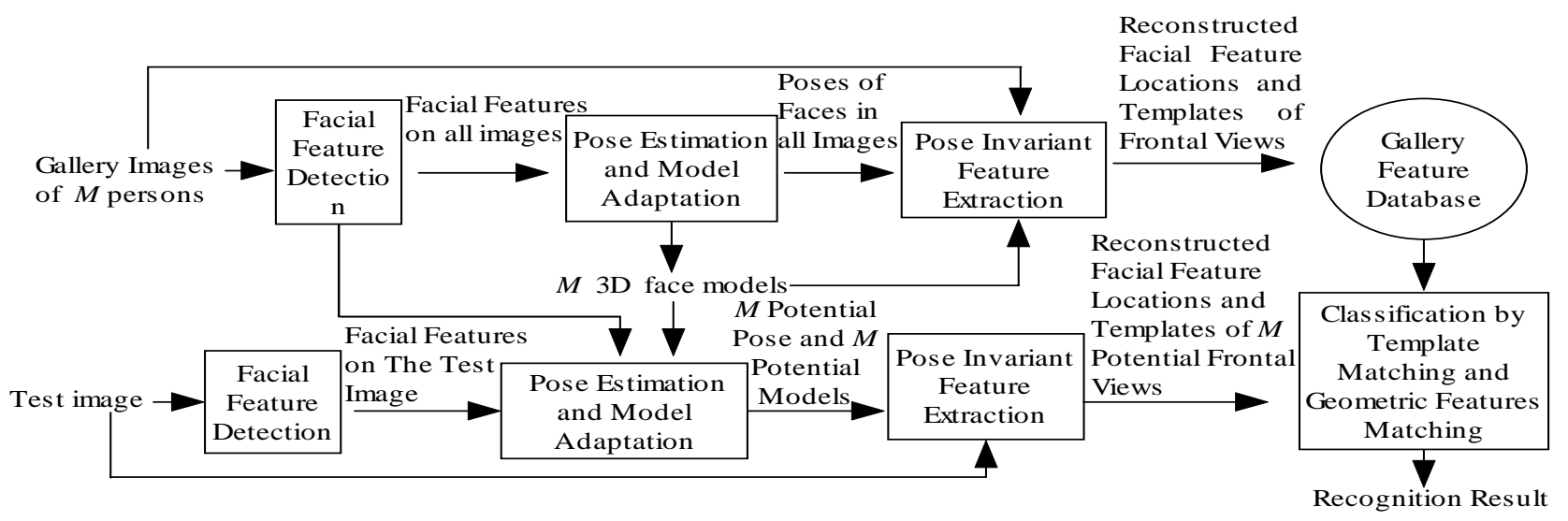

Fig. 1. Overall method architecture of the face recognition. 
model adaptation directly for these images using Lam and Yan's method and obtain a pose and a face model for each gallery face. Based on each gallery face image and the corresponding estimated face model, an efficient algorithm is proposed to estimate a pose and a face model for a test face. Thus the test face totally has $\mathrm{M}$ potential poses and potential face models, where $\mathrm{M}$ is the number of the candidates in the gallery. In the pose invariant feature extraction operation, we compute the facial feature points' locations and the facial feature templates of frontal views and potential ones from the gallery face images and the test image respectively based on the estimated poses and models. Finally, the proposed method achieves classification by comparing the obtained template and geometric features from the gallery images and the test image.

\section{Facial Feature Extraction}

Locating facial features is an important step in face recognition. In the proposed method, the rough face contour, two outside eye corners $\mathbf{p}^{(0)}$ and $\mathbf{p}^{(3)}$, two inside eye corners $\mathbf{p}^{(1)}-\mathbf{p}^{(2)}$, two mouth corners $\mathbf{p}^{(4)}-\mathbf{p}^{(5)}$, a nose tip $\mathbf{p}^{(8)}$ and tow eyebrow points $\mathbf{p}^{(6)}-\mathbf{p}^{(7)}$ (see Fig. 1(a)) are located by using Lam and Yan's method [6]. The ear points as shown in Fig. 6 are not used in most face recognition algorithms. They are important features for estimating the seesaw rotation in our algorithm. An edge map based algorithm is proposed to detect the ear points in this paper. In the following, we illustrate how the algorithm detects left ear point.

First of all, a 2D rotation transform is performed on the input face image $I$ to produce an upright face image $I_{u}$, in which the line that holds least square distances to four eye corners is parallel to horizontal axis

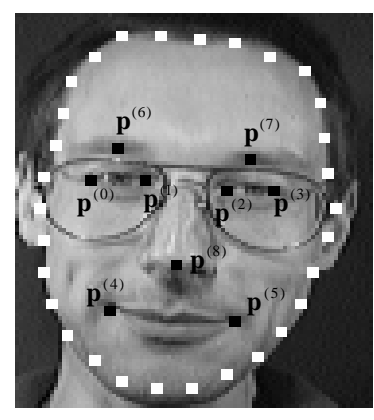

(a)

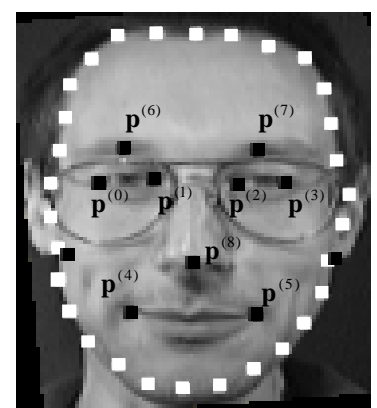

(b)

Fig. 2 Facial features in: (a) the input image $I$, (b) the upright image $I_{u}$.

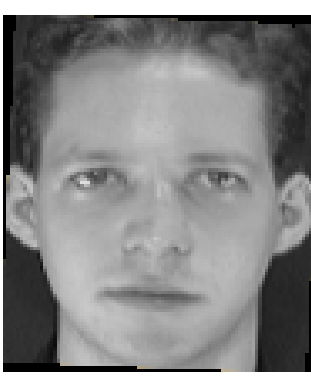

(a)

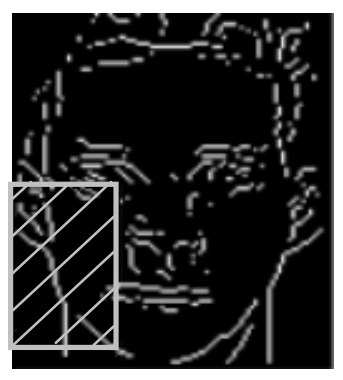

(b) (see Fig. 2(b)). The facial feature points $\mathbf{p}_{u}^{(j)}, j=0, \ldots, 8$ in $I_{u}$ correspond to $\mathbf{p}^{(j)}, j=0, \ldots, 8$ in $I$, as shown in Fig. 2(b). Then the modified canny edge detector introduced in [4] is employed to obtain the edge map E of (for example, Fig. 3(a)). From the detected facial feature points and the rough face contour in $I_{u}$, a searching region for ear points is determined in $\mathrm{E}$ as shown in Fig. 3 (b). The trivial edges in the searching region are eliminated. Canny edge detection may produce disconnected edges which correspond to the continuous contours in Iu. We develop a new edge connection operation to recover such continuity in the edge map $E$. Thus the connected edges are obtained as shown in Fig. 3 (c).

Fig. 3 (a) an upright face image. (b) The edge
map. (c) The potential face and ear boundaries.

By filtering out edges that have a nonnegative slope and retaining only the largest connected edges, we obtain the potential face and ear boundaries $\Gamma$ as shown in Fig. 3 (d). It is assumed that the outermost curve in $\Gamma$, says $\ell$ (i.e. $\overline{a d}$ in Fig. 3 (d)), should include both ear boundary and face boundary. We estimate the salient points on $\ell$ by R/J curvature based curve partition algorithm [5]. The salient points, which are inward 
bending points (illustrated in Fig. 4(a)) satisfying the condition that R/J curvature at these points are larger than a predefined threshold value, will be chosen as possible ear points. In order to exclude the false candidates such as the neck point (the joint point of the face boundary and the neck boundary), anthropocentric constraints are used to verify each possible ear point. The anthropocentric constraints are formed based on some prior knowledge and statistics obtained from some 200 face images. Let $d_{t s}$ denote the vertical distance between $\ell$ 's top end point $\mathbf{p}_{u t}$ and a possible ear point $\mathbf{p}_{u s}$, and $d_{l e m}$ denote the vertical distance between $\mathbf{p}_{u}^{(0)}$ and $\mathbf{p}_{u}^{(4)}$, see Fig. 4(b). If $d_{t s}>d_{l e m}$ or $\mathbf{p}_{u s}$ is on the right side of $\mathbf{p}_{u}^{(0)}, \mathbf{p}_{u s}$ will be rejected as the left ear point. If no candidate is viable to pass the verification, it means that no left ear point is detected; otherwise the viable candidate, which holds the largest $\mathrm{R} / \mathrm{J}$ curvature will be regarded as the detected left ear point.

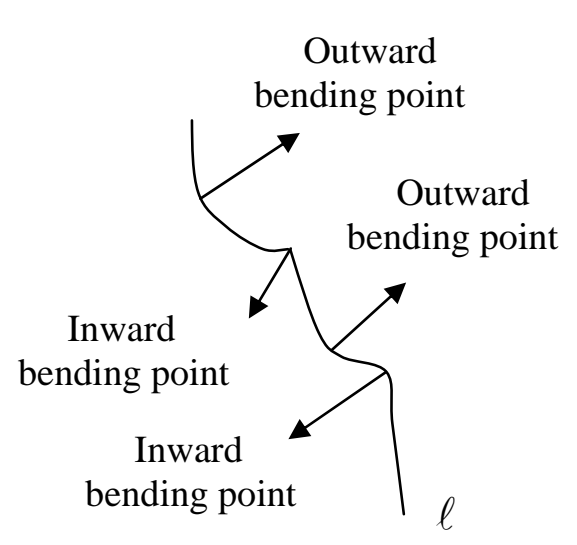

(a)

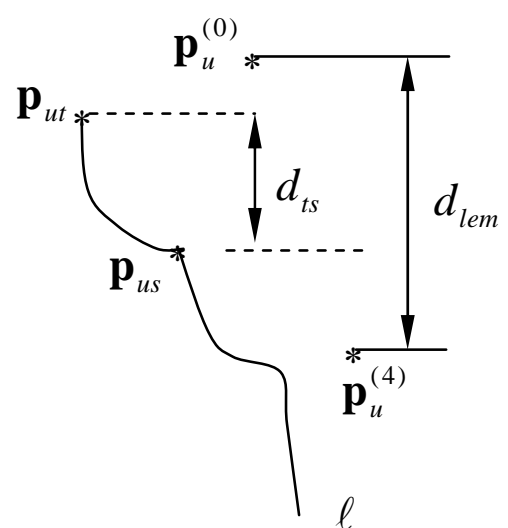

(b)

Fig. 4. (a)Bending points on the left face and ear boundary $\ell$ (b) The elements related to the anthropocentric constraints.

Similarly, we can detect the right ear point. In Iu, the left ear point and the right ear point are labelled as $\mathbf{p}_{u}^{(9)}$ and $\mathbf{p}_{u}^{(10)}$ respectively (see Fig. 2(b)). Some examples of the detected ear points are shown in Fig. 5.

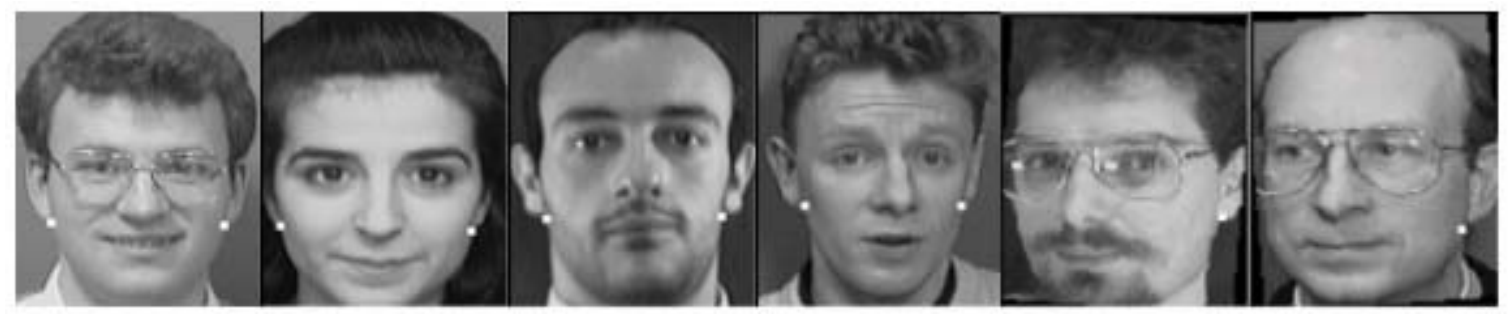

Fig. 5 The examples of ear points detected by our method.

\section{$4 \quad$ Pose Estimation and 3D Model Adaptation}

In this paper, a face image is regarded as a 2D orthogonal projection of a 3D face. While a 3D face is originally posed in the world coordinate system as shown in Fig. 6(a), its projection on the image plane will be a face image in front view. The image plane is always perpendicular to the z-axis of the word coordinate system. When the 3D face has a certain rotation around the origin of the world coordinate, its 2D projection on image plane is a face image with corresponding pose. Any rotation can be uniquely decomposed into three orderly rotations--seesaw rotation, sidespin rotation, and in image plane rotation, which are around $\mathrm{x}$ axis, y-axis and z-axis by $\theta_{x}, \theta_{y}$ and $\theta_{z}$ respectively. In this paper, all gallery images and test images are adjusted to upright face images by $2 \mathrm{D}$ rotation operation mentioned in section 2 . In addition, it is assumed that the face in each gallery image has no seesaw rotation. Thus for faces in upright gallery images, only 
small sidespin ration angles $\theta_{y-g}(m)$, for $m=1, \ldots M$ need to be estimated, where $M$ is the number of candidates in the gallery. For the face in an upright test image, we need to the estimate seesaw ration angle $\theta_{x-t}$ and the sidespin ration angle $\theta_{y-t}$. In our method, a 3D face model is used for pose estimation. The face model will be adapted to fit a particular person in the process of pose estimation.

\subsection{The adaptive face model}

A 3D adaptive model similar to that used in Lam and Yan's method (cylindrical volum with a less convex surface part as face) used to represent the 3D geometry of a head. As shown in Fig. 6(a), facial feature points on the 3D model are labeled as $\mathbf{P}^{(j)}, j=0, \ldots, 10$. The 3D model is originally located in world coordinates system under two conditions: (1) the four eye corners are coplanar on $\mathrm{x}-\mathrm{z}$ plane; (2) the $\mathrm{y}-\mathrm{z}$ plane is the symmetrical plane of the face model. Therefore we can obtain its frontal view projection on the image plane.

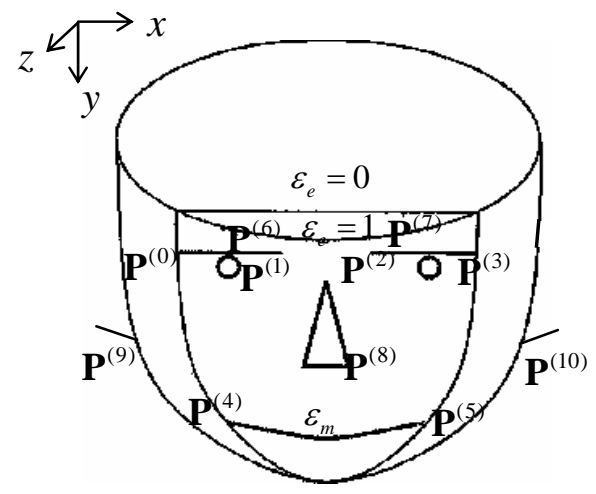

(a)

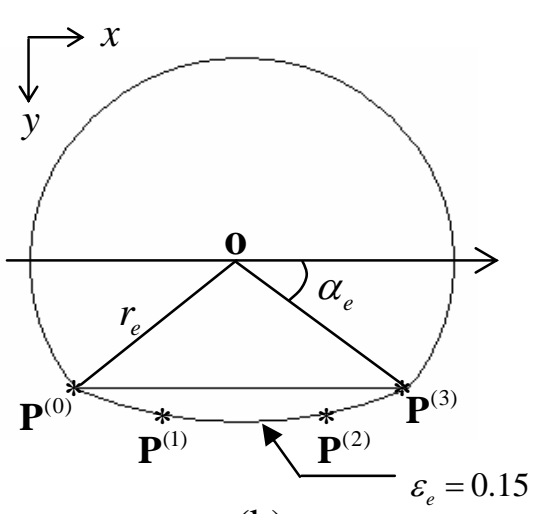

(b)

Fig. 6 (a) The 3D face model in original pose. (b) The horizontal cross-section of the face model through the eye corners.

In [6], the convexity of the less convex surface of the face model is specified by $\varepsilon$. When $\varepsilon=0$, the less convex surface area becomes a flat plane, while $\varepsilon=1$, the model is a cylinder. In our method, considering that the convexity of the surface around mouth is evidently larger than that around eyes, we use $\varepsilon_{m}$ and $\varepsilon_{e}$ to specify the local convexities of the less convex surface around the mouth and the eyes respectively. In this paper, we set $\varepsilon_{e}=0.15$, while $\varepsilon_{m}=0.85$. With fixed $\varepsilon_{e}$, the structure of the horizontal cross-section of the face model passing through four eye corners is specified by parameters $r_{e}$ and $\alpha_{e}$ (illustrated in Fig. 6(b)). The arc passing through points $\mathbf{P}^{(0)}, \mathbf{P}^{(1)}, \mathbf{P}^{(2)}$ and $\mathbf{P}^{(3)}$ is also a part of a circle. The origin of the world coordinates system is on this cross-section and is marked by $\mathbf{O}$ in Fig. 6(b). Similarly, the structure of the horizontal cross-section of the face model passing through two mouth corners is specified by parameters $r_{m}$ and $\alpha_{m}$. In addition, the information of ear points is appended in our face model with the assumption that the depth distance (along z-axis) between an ear point and an outside eye corner is $r_{e}$. Thus we adapt the simple 3D face model to fit a particular person by estimating parameters $r_{e}, \alpha_{e}, r_{m}$ and $\alpha_{m}$ from a 2D face image.

\subsection{Pose estimation and model adaptation}

Suppose there are $M$ face images in nearly front view in the gallery, one image per candidate. With the assumption that the face in each gallery image has no seesaw rotation, we can estimate $\theta_{y-g}(m), r_{e-g}(m)$, $\alpha_{e-g}(m), r_{m-g}(m)$, and $\alpha_{m-g}(m), m=1, \ldots, M$ from each gallery face image using Lam and Yan's method. These 5 parameters specify the 3D model which fits the face in the $m$-th gallery image. The facial feature points in the $m$-th gallery face image are represented by $\mathbf{p}_{u-g}^{(m, j)}$, corresponding to $\mathbf{p}_{u}^{(j)}$ on Fig. 2(b), $j=1, \ldots, 10$. 


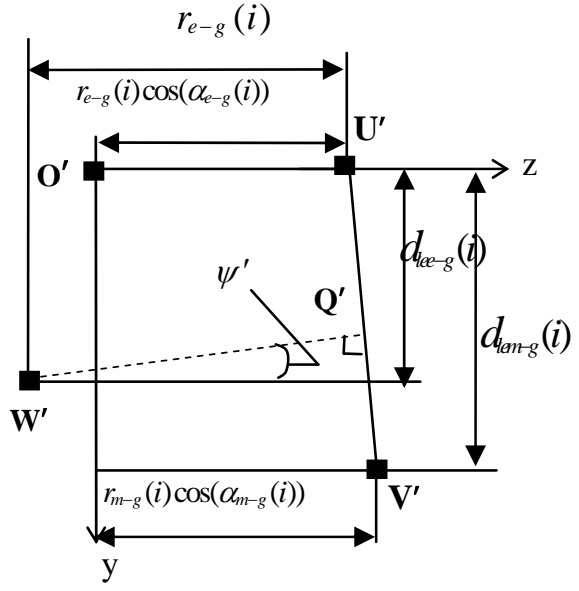

(a)

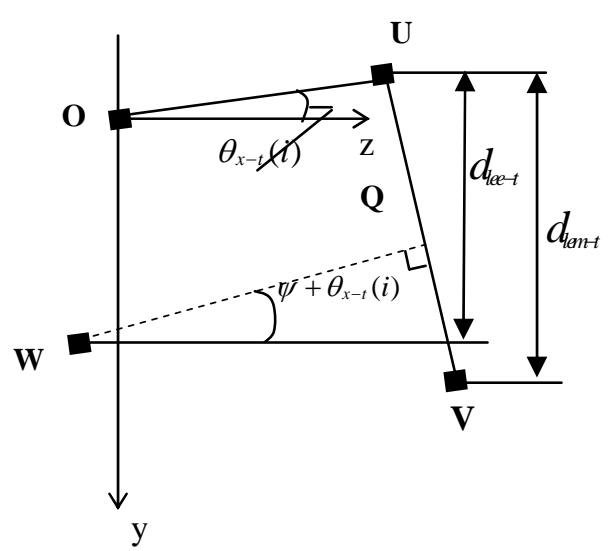

(b)

Fig.7. The projection of the left eye corner, the left ear point and the left mouth corner on $y-z$ plan (when $\theta_{y-t}=0^{\circ}$ and $\theta_{y-g}(i)=0^{\circ}$ ), for (a) the test face (b) the $i$-th gallery face.

For a test image, we propose a new algorithm to estimate $\theta_{x-t}$ and $\theta_{y-t}$, and to achieve 3D face model adaptation by estimating parameters $r_{e-t}, \alpha_{e-t}, r_{m-t}$, and $\alpha_{m-t}$. Let $\mathbf{p}_{u-t}^{(j)}, j=1, \ldots, 10$ represent the facial feature points in the test face image. Assuming that the test image is a face image of the $i$-th $(i \in[1, M])$ candidate in the gallery, we first estimate the corresponding potential seesaw rotation angle $\theta_{x-t}(i)$. As the test face is not surly same as the $i$-th gallery face, $\theta_{x-t}(i)$ is a potential $\theta_{x-t}$. Suppose that both the test face and the $i$-th gallery face have no sidespin rotation $\theta_{y-t}=0^{\circ}$ and $\theta_{y-g}(i)=0^{\circ}$. The left ear point, the left outside eye point and the left mouth corner on the test face are projected on y-z plane, and the projections of these facial feature points are denoted by $\mathrm{W}, \mathrm{U}$ and V respectively as shown in Fig. 7(a). The corresponding facial feature points on the $i$-th gallery face are also also projected on $y$-z plane, and the projections of these three facial feature points are denoted by $\mathbf{W}^{\prime}, \mathbf{U}^{\prime}$ and $\mathbf{V}^{\prime}$ respectively as shown in Fig. 7(b). Line segments $\mathbf{W Q}$ and $\mathbf{W}^{\prime} \mathbf{Q}^{\prime}$ are perpendicular to $\mathbf{U} \mathbf{V}$ and $\mathbf{U}^{\prime} \mathbf{V}^{\prime}$, with intersection $\mathbf{Q}$ and $\mathbf{Q}^{\prime}$. Thus we have following equations:

$$
\begin{aligned}
& \left(|\overline{\mathbf{W Q}}| \operatorname{tg}\left(\psi+\theta_{x-t}(i)\right)+|\overline{\mathbf{Q U}}|\right) /|\overline{\mathbf{U V}}|=d_{\text {lee- }} / d_{\text {lem-t }}, \\
& \left(\left|\overline{\mathbf{W}^{\prime} \mathbf{Q}^{\prime}}\right| \operatorname{tg}\left(\psi^{\prime}\right)+\left|\overline{\mathbf{Q}^{\prime} \mathbf{U}^{\prime}}\right|\right) /\left|\overline{\mathbf{U}^{\prime} \mathbf{V}^{\prime}}\right|=d_{\text {lee-g }}(i) / d_{\text {lem-g}}(i)
\end{aligned}
$$

where $d_{\text {lee- }}$ denotes the vertical distance between the left out side eye corner $\mathbf{p}_{u-t}^{(0)}$ and the left ear point $\mathbf{p}_{u-t}^{(9)}$ on the test image, $d_{l e m-t}$ denotes the vertical distance between $\mathbf{p}_{u-t}^{(0)}$ and the left mouth corner $\mathbf{p}_{u-t}^{(4)}, d_{l e e-g}(i)$ denotes the vertical distance between the $\mathbf{p}_{u-g}^{(i, 0)}$ and $\mathbf{p}_{u-g}^{(i, 9)}$ on the $i$-th gallery image, and $d_{l e m-g}$ denotes the vertical distance between the $\mathbf{p}_{u-g}^{(i, 0)}$ and $\mathbf{p}_{\mu \rightarrow g}^{(i, 4)}$. As it is assumed that the test face and the $\mathrm{i}$-th gallery face belong to the same person, we have $|\mathbf{W Q}|=k\left|\mathbf{W}^{\prime} \mathbf{Q}^{\prime}\right|,|\mathbf{Q U}|=k\left|\mathbf{Q}^{\prime} \mathbf{U}^{\prime}\right|, \psi=\psi^{\prime}$, and $|\mathbf{U V}|=k\left|\mathbf{U}^{\prime} \mathbf{V}^{\prime}\right|$. The scaling factor $\mathrm{k}$ is used to remove the size difference of the test face and the $i$-th gallery face. Thus the corresponding potential seesaw rotation angle of the test face can be formulated as follows:

$\theta_{x-t}(i)=\tan ^{-1}\left(\left(\frac{d_{l e e-g}(i) / d_{l e n-g}(i)}{d_{l e-t} / d_{e n-t}}\left(\left|\overline{\mathbf{W}^{\prime} \mathbf{Q}^{\prime}}\right| \operatorname{tg}\left(\psi^{\prime}\right)+\left|\overline{\mathbf{Q}^{\prime} \mathbf{U}^{\prime}}\right|\right)-\left|\overline{\mathbf{Q}^{\prime} \mathbf{U}^{\prime}}\right|\right) /\left|\overline{\mathbf{W}^{\prime} \mathbf{Q}^{\prime}}\right|\right)-\psi^{\prime}$.

Based on the $i$-th adapted gallery face model and facial feature points on the $i$-th gallery image, $\overline{\mathbf{W}^{\prime} \mathbf{Q}^{\prime}}$, $\mathbf{U}^{\prime} \mathbf{V}^{\prime}$ and $\psi^{\prime}$ can be computed by following equations:

$\psi^{\prime}=\tan ^{-1}\left(\left(r_{m-g}(i) \sin \left(\alpha_{m-g}(i)\right)-r_{e-g}(i) \sin \left(\alpha_{e-g}(i)\right) / d_{l e m-g}(i)\right)\right.$,

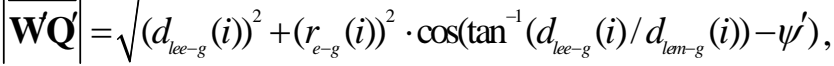

$\left|\overline{\mathbf{Q}^{\prime} \mathbf{U}^{\prime}}\right|=\sqrt{\left(d_{\text {lee-g }}(i)\right)^{2}+\left(r_{e-g}(i)\right)^{2}} \cdot \sin \left(\tan ^{-1}\left(d_{l e-g}(i) / d_{l e m-g}(i)\right)-\psi^{\prime}\right)$

From (3)-(6), the corresponding seesaw rotation angle $\theta_{x-t}(i)$ can be computed. As $d_{l e e-g}(i) / d_{l e m-g}(i)$, $d_{l e e-t} / d_{l e m-t}$ as well as the parameters of the $i$-th gallery model are independent to $\theta_{y-t}$ and $\theta_{y-g}(i)$, the 
proposed algorithm is available to compute $\theta_{x-t}(i)$ for any $\theta_{y-t}$ and $\theta_{y-g}(i)$ (without the requirement of $\theta_{y-t}=0^{\circ}$ and $\theta_{y-g}(i)=0^{\circ}$ ). In the same way, another corresponding potential seesaw rotation angle for the test face can be computed using the information provided in the right feature points. We average these two corresponding potential seesaw rotation angles as the final $\theta_{x-t}(i)$. In this paper, we assume that the seesaw rotation angle of the test face is in range of $-25^{\circ}$ to $+25^{\circ}$. Thus if $\theta_{x-t}(i)>25^{\circ}$ or $\theta_{x-t}(i)<-25^{\circ}$, it will be set at $25^{\circ}$ or $-25^{\circ}$ respectively. If we do not detect any ear point on the $i$-th gallery face image or on the test face image, then $\theta_{x-t}(i)$ is assigned to be zero.

Based on the estimated $\theta_{x-t}(i)$, we can further estimate corresponding, and $r_{e-t}(i), \alpha_{e-t}(i)$ and $\theta_{y-t}(i)$ using the geometric information about two outside eye corners and the face contour in the test face image. Let $\Pi_{e i}$ represent a circle that passes through the outside eye corners on the corresponding potential model of the test face and centers at the origin of the word coordinate system. Let $\Theta_{e i}$ be the projection of $\Pi_{e i}$ on the image plane. If $\theta_{x-t}(i)=0^{\circ}, \Theta_{e i}$ should be a line passing the outside eye corners $\mathbf{p}_{u-t}^{(0)}$ and $\mathbf{p}_{u-t}^{(3)}$ in the test image (Lam and Yan's algorithm just consider this situation), otherwise $\Theta_{e i}$ should be an ellipse passing the outside eye corners in the test image as shown in Fig. 8. $\mathbf{p}_{u-t}^{(11)}\left(x_{p_{u-1}^{(u+1}}, y_{p_{u-1}^{(u+1}}\right)$ represents the middle point between $\mathbf{p}_{u-t}^{(0)}$ and $\mathbf{p}_{u-t}^{(3)}$. In this paper, we assume that $\mathbf{p}_{u-t}^{(0)}$ and $\mathbf{p}_{u-t}^{(3)}$ are not occluded in an image. $\mathbf{p}_{a i}\left(x_{p_{a i}}, y_{p_{a i}}\right)$ and $\mathbf{p}_{b i}\left(x_{p_{u}}, y_{p_{u t}}\right)$ represent the two end points of the long axis of the ellipse $\Theta_{e i}$ respectively. $\mathbf{p}_{c i}\left(x_{p_{i}}, y_{p_{i d}}\right)$ is the center of $\Theta_{e i}$.

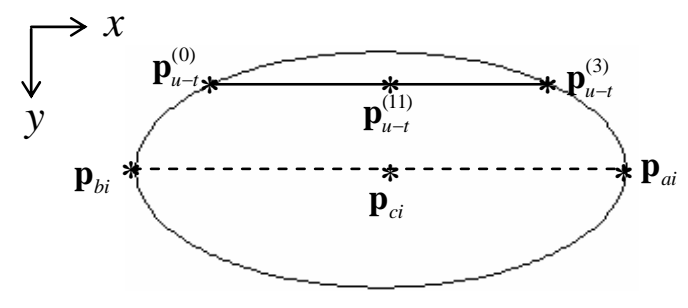

(a)

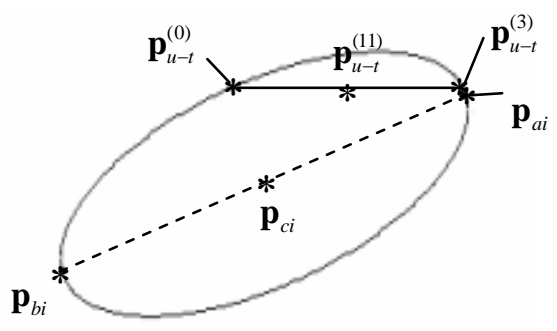

(b)

Fig.8 $\Theta_{e i}$, the projection of $\Pi_{e i}$ in test image when $\theta_{x-t}(i)>0^{\circ}$ and (a) $\theta_{y-t}(i)=0^{\circ}$, (b) $\theta_{y-t}(i)>0^{\circ}$.

The ellipse $\Theta_{e i}(x, y)$ in the test image can be formulated as follows:

$x=r_{e-t}(i) \cos \left(\theta_{y-t}(i)\right) \cos \lambda+r_{e-t}(i) \sin \left(\theta_{y-t}(i)\right) \cos \left(\theta_{x-t}(i)\right) \sin \lambda+x_{p_{c}}$

$$
y=-r_{e-t}(i) \sin \left(\theta_{x-t}(i)\right) \sin \lambda+y_{p_{c i}}
$$

where $\lambda \subset(0,2 \pi)$ is the independent variable and $\left(x_{p_{i}}, y_{p_{i}}\right)$ denotes the location of the ellipse's center. $r_{e-t}(i), x_{p_{d}}$ and $y_{p_{i}}$ are given by:

$r_{e-t}(i)=\left|\mathbf{p}_{u-t}^{(0)}-\mathbf{p}_{u-t}^{(3)}\right| /\left(2 \cos \left(\theta_{y-t}(i)\right) \cos \alpha_{e-t}(i)\right)$

(9)

$x_{p_{s}}=-\tan \left(\theta_{y-t}(i)\right) \tan \left(\alpha_{e-t}(i)\right) \cos \left(\theta_{x-t}(i)\right)\left(\left|\mathbf{p}_{u-t}^{(0)}-\mathbf{p}_{u-t}^{(3)}\right| / 2\right)+x_{p_{u-t}^{(1)}}$

$(10)$

$y_{p_{a}}=\tan \left(\alpha_{e-t}(i)\right) \sin \left(\theta_{x-t}(i)\right)\left|\mathbf{p}_{u-t}^{(0)}-\mathbf{p}_{u-t}^{(3)}\right| /\left(2 \cos \left(\theta_{y-t}(i)\right)\right)+y_{p_{u-t}^{(i)}}$.

Combining (9), (10) and (11), we rewrite (7) and (8) as follows:

$$
\begin{aligned}
x= & \cos \lambda \cdot\left|\mathbf{p}_{u-t}^{(0)}-\mathbf{p}_{u-t}^{(3)}\right| /\left(2 \cos \left(\alpha_{e-t}(i)\right)\right)+\tan \left(\theta_{y-t}(i)\right) \cos \left(\theta_{x-t}(i)\right) \sin \lambda \cdot\left|\mathbf{p}_{u-t}^{(0)}-\mathbf{p}_{u-t}^{(3)}\right| /\left(2 \cos \left(\alpha_{e-t}(i)\right)\right) \\
& -\tan \left(\theta_{y-t}(i)\right) \tan \left(\alpha_{e-t}(i)\right) \cos \left(\theta_{x-t}(i)\right)\left(\left|\mathbf{p}_{u-t}^{(0)}-\mathbf{p}_{u-t}^{(3)}\right| / 2\right)+x_{p_{u-t}^{(1) 1}}
\end{aligned}
$$

$$
y=-\sin \left(\theta_{x-t}(i)\right) \sin \lambda \cdot\left|\mathbf{p}_{u-t}^{(0)}-\mathbf{p}_{u-t}^{(3)}\right| /\left(2 \cos \left(\theta_{x-t}(i)\right) \cos \left(\alpha_{e-t}(i)\right)\right)+\tan \left(\alpha_{e-t}(i)\right) \sin \left(\theta_{x-t}(i)\right)\left|\mathbf{p}_{u-t}^{(0)}-\mathbf{p}_{u-t}^{(3)}\right| /\left(2 \cos \left(\theta_{y-t}(i)\right)\right)+y_{p_{u-t}^{(1) 1}}
$$


Then we try to locate $\mathbf{p}_{a i}$ and $\mathbf{p}_{b i}$ on $\Theta_{e i}$ on the image plane. One of $\mathbf{p}_{a i}\left(x_{p_{u}}, x_{p u}\right)$ and $\mathbf{p}_{b i}\left(x_{p_{u}}, x_{p_{u}}\right)$ is represented by symbol $\mathbf{p}_{i}\left(x_{p_{i}}, x_{p_{i}}\right)$. We have:

$$
\begin{aligned}
& x_{p_{i}}=r_{e-t}(i) \cos \left(\theta_{y-t}(i)\right) \cos \lambda_{p_{i}}+r_{e-t}(i) \sin \left(\theta_{y-t}(i)\right) \cos \left(\theta_{x-t}(i)\right) \sin \lambda_{p_{i}}+x_{p_{c i}}, \\
& y_{p_{i}}=-r_{e-t}(i) \sin \left(\theta_{x-t}(i)\right) \sin \lambda_{p_{i}}+y_{p_{d i}}, \\
& \left(x_{p_{i}}-x_{p_{d i}}\right)^{2}+\left(y_{p_{i}}-y_{p_{c i}}\right)^{2}=\left(r_{e-t}(i)\right)^{2} .
\end{aligned}
$$

Thus the following equation can be conduced from (14), (15) and (16):

$\left(\cos \left(\theta_{x-t}(i)\right) \cos \left(\theta_{y-t}(i)\right)\right)^{2}\left(\tan \left(\lambda_{p_{i}}\right)\right)^{2}-\sin \left(2 \theta_{y-t}(i)\right) \cos \left(\theta_{x-t}(i)\right) \tan \left(\lambda_{p_{i}}\right)-\left(\sin \left(\theta_{y-t}(i)\right)\right)^{2}=0$.

$\tan \left(\lambda_{p_{i}}\right)=\tan \left(\left(\theta_{y-t}(i)\right) / \cos \left(\theta_{x-t}(i)\right), \lambda_{p_{i i}}=\tan ^{-1}\left(\tan \left(\left(\theta_{y-t}(i)\right) / \cos \left(\theta_{x-t}(i)\right)\right)\right.\right.$ and $\lambda_{p_{x}}=\tan ^{-1}\left(\tan \left(\left(\theta_{y-t}(i)\right) / \cos \left(\theta_{x-t}(i)\right)\right)+\pi\right.$ are hence obtained from (17). Referring (12) and (13), we found that the pair of locations of $\mathbf{p}_{a i}$ and $\mathbf{p}_{b i}$ in image plane are determined by $\alpha_{e-t}(i)$ and $\theta_{y-t}(i)$. According to statistics obtained from some 100 face images, $\alpha_{e-t}(i)$ should be in range of $35^{\circ}$ to $65^{\circ}$. In this paper we assume $\theta_{y-t}(i) \in\left[-30^{\circ}, 30^{\circ}\right]$. Thus based on dense sampling $\alpha_{e-t}(i) \in\left[35^{\circ}, 65^{\circ}\right]$ and $\theta_{y-t}(i) \in\left[-30^{\circ}, 30^{\circ}\right]$, we can compute K possible pairs of $\mathbf{p}_{a i}$ and $\mathbf{p}_{b i}$ which are represented by $\Phi_{k}\left(\mathbf{p}_{a i}(k), \mathbf{p}_{b i}(k)\right), k=1, \ldots, K$. In the following, $\Phi_{k}, k=1, \ldots, K$ will be used to approximate all possible pairs of $\mathbf{p}_{a i}$ and $\mathbf{p}_{b i}$ for $\alpha_{e-t}(i) \in\left[35^{\circ}, 65^{\circ}\right]$ and $\theta_{y-t}(i) \in\left[-30^{\circ}, 30^{\circ}\right]$. Let $\alpha_{e-t}(i, k)$, $\theta_{y-t}(i, k)$ represent the sample values of $\alpha_{e-t}(i)$ and $\theta_{y-t}(i)$ corresponding to the $\Phi_{k}$. The smaller the sampling interval is, the larger $\mathrm{K}$ is and the more efficient the approximation is. For our application, we set the interval at $1^{\circ}$ for sampling both $\alpha_{e-t}(i)$ and $\theta_{y-t}(i)$, so as a result $\mathrm{K}$ is equal to 1800 . According to the geometric character of our face model, it is given that among all possible pairs of $\mathbf{p}_{a i}$ and $\mathbf{p}_{b i}$, only the true one will appear on the face contour in the test image and the true one will definitely appear on the face contour. Let $d_{c p_{p i}}(k)$ represent the shortest distance from $\mathbf{p}_{a i}(k)$ to the detected face contour and $d_{c p_{i v}}(k)$ represent the shortest distance from $\mathbf{p}_{b i}(k)$ to the detected face contour. $d_{c p_{i}}(k)$ is the sum of $d_{c p_{a i}}(k)$ and $d_{c p_{i v}}(k)$. Then we estimate $\mathbf{p}_{a i}$ and $\mathbf{p}_{b i}, r_{e-t}(i), \alpha_{e-t}(i)$ and $\theta_{y-t}(i)$ by $\mathbf{p}_{a i}(c), \mathbf{p}_{b i}(c)$, $\left(\left|\mathbf{p}_{a i}(c)-\mathbf{p}_{b i}(c)\right|\right) / 2, \alpha_{e-t}(i, c)$ and $\theta_{y-t}(i, c)$ respectively, where the $d_{c p_{i}}(c)$ is the smallest one among $d_{c p_{i}}(k), k=1, \ldots, K, 1 \leq c \leq K$. We can estimate the corresponding $r_{m-t}(i), \alpha_{m-t}(i)$ and $\theta_{y-t}(i)$ in the same way but using the geometric information about two outside mouth corners and the face contour in the test face image. We average the two potential sidespin rotation angles estimated by using the eye corners information and the mouth corners information respectively as the final potential sidespin rotation angle $\theta_{y-t}(i)$.

In this paper, the proposed algorithm is employed to estimates the pose and the 3D face model of the test face, assuming that the test image is an image of the $m$-th candidate in the gallery for $m=1, \ldots M$. Thus we obtain $M$ potential poses and $M$ potential models for the test face image referring to all candidates in the gallery. Among these potential poses, the $i$-th one will be regarded as the matched potential pose, if the test face and the $i$-th gallery face really belong to one person. The matched potential pose consists of matched potential seesaw rotation angle $\theta_{y m-t}$ and matched potential sidespin rotation angle $\theta_{y m-t}$.

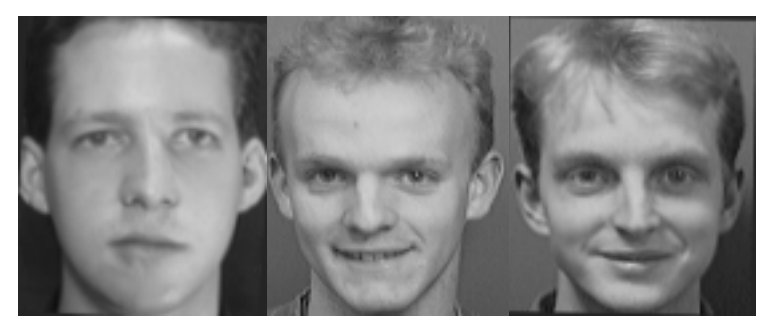

$\left(\theta_{y-t}=1.4^{\circ}, \theta_{x-t}=0^{\circ}\right)\left(\theta_{y-t}=-3.1^{\circ}, \theta_{x-t}=0^{\circ}\right)\left(\theta_{y-t}=0.8^{\circ}, \theta_{x-t}=0^{\circ}\right)$

\section{(a)}

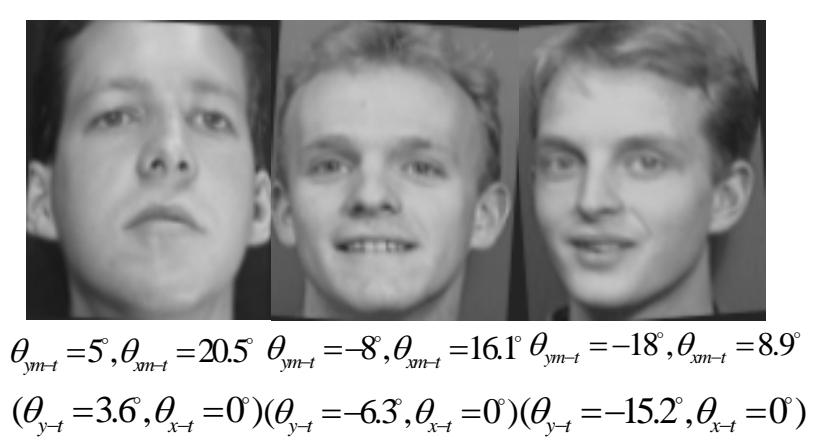

(b)

Fig.9 Some test faces in (b) and their matched potential poses obtained by the proposed algorithm referring to the corresponding gallery faces in (a). The poses of the test faces and the gallery faces estimated by Lam and Yan's algorithm are also given in parentheses for comparison.

Fig. 9 shows some examples to illuminate the performance of the proposed algorithm for pose estimation. The images in Fig. 9.(a) show some test faces and the images in Fig. 9.(b) show the gallery faces belonging 
to the same person. The matched potential poses of these test faces, which are estimated by the proposed algorithm, are shown in Fig. 9(b). For comparison, the poses of these test faces and gallery faces estimated by Lam and Yan's pose estimation algorithm [6] are also given in the parentheses. The result shows that the proposed pose estimation algorithm can obtain effective and general estimate of poses (including both seesaw and sidespin rotations) for test faces.

\section{Pose Invariant Feature Extraction and Classification}

Based on estimated pose and 3D face model of each gallery faces, we reconstruct a set of facial feature points' locations $\mathbf{p}_{u s-g}^{(m, j)}\left(x_{p_{w-q}}(m, j), y_{p_{w-\vartheta}}(m, j)\right), j=1, \ldots, 8$, on the frontal view of each gallery face from $\mathbf{p}_{u-g}^{(m, j)}\left(x_{p_{u s g}}(m, j), y_{p_{u-g}}(m, j)\right), j=1, \ldots, 8$, for $m=1, \ldots, M$, using affine transform. Similarly, based on the $M$ potential poses and corresponding potential models of the test face, $M$ sets of facial feature points' locations $\mathbf{p}_{u s-t}^{(m, j)}\left(x_{p_{w-t}}(m, j), y_{p_{w-t}}(m, j)\right), j=1, \ldots, 8, m=1, \ldots, M$ on the corresponding $M$ potential frontal views of the test face are reconstructed from $\mathbf{p}_{u-t}^{(j)}\left(x_{p_{u-1}}(j), y_{p_{u-t}}(j)\right), j=1, \ldots, 8$. For $m=1, \ldots, M$, point set $\mathbf{p}_{u s-t}^{(m, j)}, j=1, \ldots, 8$ are normalized and aligned to $\mathbf{p}_{u s-g}^{(m, j)}, j=1, \ldots, 8$, besides $r_{e-t}(m)$ and $r_{m-t}(m)$ are normalized to $r_{e-g}(m)$ and $r_{m-g}(m)$. We define $D_{w}(m), m=1, \ldots M$ as follows:

$D_{w}(m)=\sum_{j=0}^{8} k_{j}\left|\mathbf{p}_{u s-t}^{(m, j)}-\mathbf{p}_{u s-g}^{(m, j)}\right|+k_{9}\left|r_{e-t}(m)-r_{e-g}(m)\right|+k_{10}\left|r_{m-t}(m)-r_{m-g}(m)\right|$.

Corresponding geometric features matching is then preformed by computing $D_{w}^{m}$, for $m=1, \ldots, M$ to measure the similarity between the test face and each gallery face. $N(N \leq M)$ faces in the gallery which satisfy $D_{w}^{m} \leq \delta_{D}$ ( $\delta_{D}$ is a threshold) are chosen as qualified gallery faces and passed on for the further recognition process. Corresponding to these $\mathrm{N}$ qualified faces in the gallery, $\mathrm{N}$ potential poses and potential models are remained for the test face. Based on the $n$-th remained potential pose and corresponding potential model, for $n=1, \ldots, N$, the eyes and eyebrows template $T_{e-t}(n)$, the nose template $T_{n-t}(n)$ and the mouth template $T_{m-t}(n)$ in the $n$-th potential frontal view of the test face are synthesized by affine transform. We also synthesized the eyes and eyebrows template $T_{e-g}(n)$, the nose template $T_{n-g}(n)$ and the mouth template $T_{m-g}(n)$ in the frontal view of the $n$-th qualified gallery face, for $n=1, \ldots, N$. Then $T_{e-t}(n), T_{n-t}(n)$ and $T_{m-t}(n)$ compared to $T_{e-g}(n), T_{n-g}(n)$ and $T_{m-g}(n)$ by correlations, for $n=1, \ldots, N$. The larger the correlations are, the more similar to the test face the corresponding qualified face in the gallery is. This comparison is referred to as corresponding template matching. Combining the results of the corresponding geometric feature matching and corresponding template matching, we can achieve final classification and find a qualified face in the gallery which is most like the test face.

In this section, the operations of normalization, alignment, and correlation in our method are same as those in Lam and Yan's method. In our method, the operations of synthesizing facial feature points' locaitons on frontal view and synthesizing facial feature templates in frontal view are similar to corresponding operations in Lam and Yan's method except for considering extra estimated seesaw rotation. In the operation of geometric feature matching, we use 9 facial feature points and 2 estimated model parameters to replace the 15 facial feature points used in Lam and Yan's method, because the 6 facial feature points on the face contour are not pose invariant features in our case when the seesaw rotation is considered. The implementation of these operations can be referenced to [6].

Fig. 10 (a) and (b) show a test face image and a gallery face image of the same person respectively. Fig. 10 (c) and (e) show facial feature templates of the test face and the gallery face synthesized by using Lam and Yan's method. Fig. 10(d) shows facial feature templates of the test face synthesized by using our method based on the matched potential pose and the matched potential face model. Compared to the facial feature templates shown in Fig. 10(c), the facial feature templates shown in Fig. 10(d) are more like in frontal view and have more similarity to those shown in Fig. 10(e).

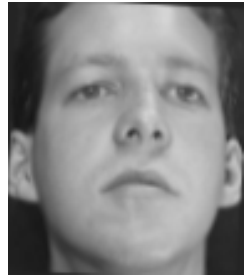

(a)

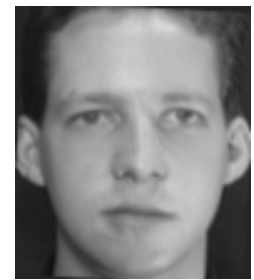

(b)

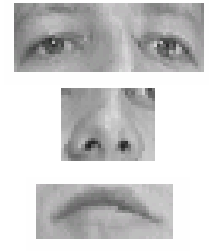

(c)

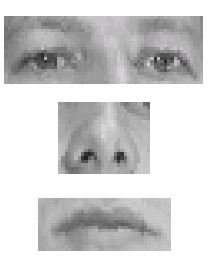

(d)

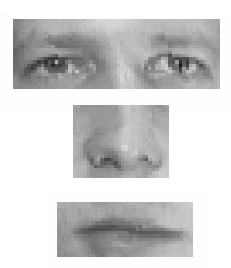

(e)

Fig.10. (a) A test face image. (b) The corresponding gallery face image which captures the same person as the test face image. The facial feature templates of the test face synthesized using Lam's method (c), of the test face synthesized using our method based on the matched potential pose (d), and of the gallery face svnthesized using Lam and Yan's method (e). 


\section{Experimental Results}

\subsection{Testing the proposed algorithm for detecting ear points}

This section gives the testing of the proposed algorithm for detecting ear points using 280 face images in ORL database (accessible at http://www.cam-orl.co.uk/ facedatabase .html). We first manually marked the ear points on each of these test images. Totally there are 453 visible ear points on these face images. The computed ear points on these images were obtained by the proposed algorithm. Our algorithm detected 378 ear points on 231 face images, and detected no ear points on the other 49 images. The average distance $D_{m c}$ between each of the 378 computed ear points and its corresponding manually marked one is 1.88 pixels.

\subsection{Evaluating the proposed pose estimation algorithm using synthetic data}

We generate random 3D synthetic faces (see example in Fig. 11.) of different particular persons using commercial software "FaceGen Modller 3.0", which is developed by Singular Inversion Inc. (http://www.facegen.com). The 3D synthetic face is then normalized according to the distance of the two outside eye corners and thus with the assumption that the projection system used here is an orthogonal projection system, the distance of the two eye corners in the frontal view projection is fixed at 200 pixels.

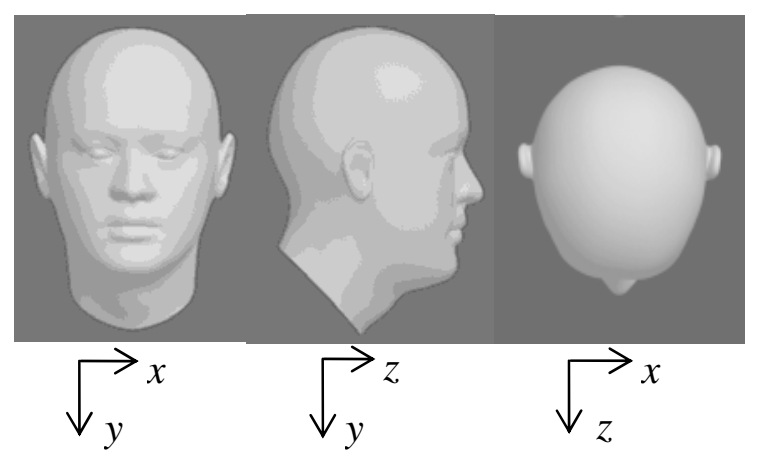

Fig. 11. Two examples of synthetic face generated by “FaceGen Modller 3.0”.

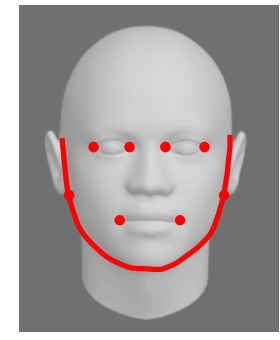

(a)

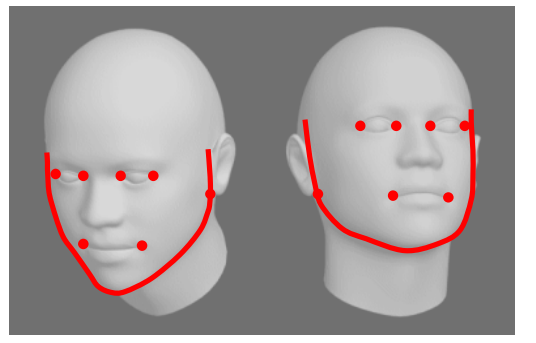

(b)
Fig. 12. Orthogonal projections of rotated synthetic face with marked facial feature points and face contours. (a) gallery data (b) test data.

We examined the performance of the pose estimation algorithm for a test image under the condition that we have the same candidate's nearly frontal view image in the gallery. 20 synthetic faces were randomly generated. Each synthetic face was first rotated with a uniformly distributed random rotation parameter set $\left(\theta_{x-g}=0^{\circ}, \theta_{y-g} \in\left[-5^{\circ}, 5^{\circ}\right], \theta_{z-g}=0^{\circ}\right)$, and the face image projected from the rotated synthetic face was used as its gallery image. Similarly we generated 6 test images for each synthetic face with 6 uniformly distributed random rotation parameter sets $\left(\theta_{x-t}(i) \in\left[-25^{\circ}, 2^{\circ}\right], \theta_{y-t}(i) \in\left[-30^{\circ}, 30^{\circ}\right], \theta_{z-t}(i)=0^{\circ}\right), i=1,2 \ldots, 6$. The facial feature points and face contours of gallery images and test images are marked manually, as shown in Fig. 12. In order to simulate the facial feature points extraction error, Gaussian random noise with standard variance $\sigma_{2 d}$ was added on the x-and y-coordinates of the marked facial feature points in both gallery data and test data. Using the proposed pose estimation algorithm, we recover the pose of each test face $\left(\theta_{x-t}{ }^{\prime}(i), \theta_{y-t}{ }^{\prime}(i)\right)$, $i=1,2, \ldots, 6$. The absolute seesaw rotation error and the absolute sidespin rotation error defined as: $\Delta \theta_{\text {xerr }}=1 / 2\left(\sum_{i=1}^{6}\left(\left|\theta_{x-t}^{\prime}(i)-\theta_{x-t}(i)\right|\right)\right)$ and $\Delta \theta_{\text {yerr }}=1 / 2\left(\sum_{i=1}^{6}\left(\left|\theta_{y-t}^{\prime}(i)-\theta_{y-t}(i)\right|\right)\right)$ were utilized to measure the accuracy of the recovered poses. Finally, the average $\Delta \bar{\theta}_{\text {xer }}$ and $\Delta \bar{\theta}_{\text {yer }}$ for 20 synthetic faces were calculated. The performance of the proposed pose estimation for test images with different $\sigma_{2 d}$ is shown in Fig. 13. For comparison, we recovered the sidespin rotations of all test images using Lam and Yan's algorithm under the same condition and then computed the average absolute sidespin rotation error which is shown in Fig. 13 (b).

From Figs. 13(a) and (b), we observe that $\Delta \bar{\theta}_{\text {xerr }}$ and $\Delta \bar{\theta}_{\text {yerr }}$ increase with the increase of $\sigma_{2 d}$. For our proposed pose estimation algorithm, they are smaller than $5.4^{\circ}$ and $6.5^{\circ}$ respectively when $\sigma_{2 d} \leq 4$. It is found that even when $\sigma_{2 d}=0, \Delta \bar{\theta}_{\text {xerr }}$ and $\Delta \bar{\theta}_{\text {yer }}$ are not equal to zero. Such systematic error is produced mainly because of the structural difference between the synthetic faces and the simple adaptive face model used in our algorithm. Sidespin rotation estimation is not independent to seesaw rotation estimation and our 
algorithm can get a better seesaw rotation estimation, so the performance of the sidespin rotation estimation in our algorithm is better than that in Lam and Yan's, see Fig. 13(b). Although the proposed pose estimation algorithm used in our face recognition method cannot estimate the pose with very good accuracy, it is more general and is more efficient to be used to improve the face recognition result compared with the pose estimation algorithm used in Lam and Yan's method, as illuminated in section 6.3.

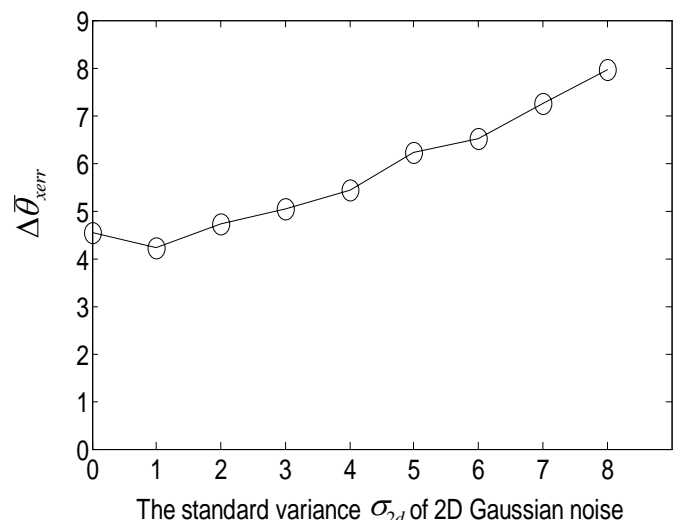

(a)

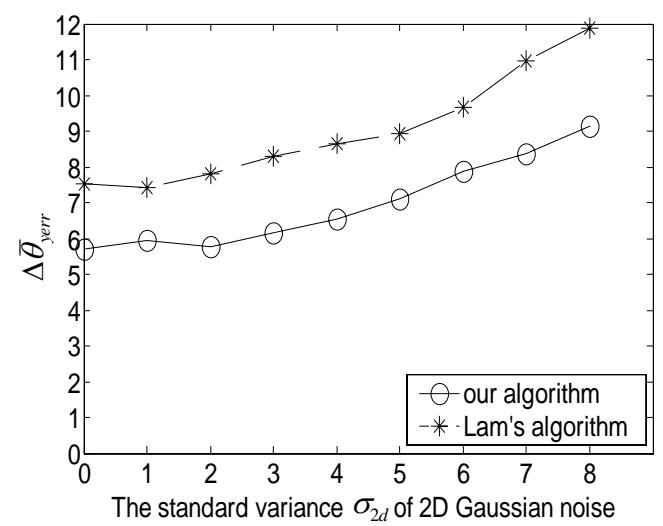

(b)

Fig. 13. Performance of pose estimation under different 2D noise on measurement of marked facial feature points: (a) $\Delta \bar{\theta}_{\text {xerr }}$ by using the proposed pose estimation algorithm; (b) $\Delta \bar{\theta}_{\text {yerr }}$ by using the proposed pose estimation algorithm and by using the pose estimation algorithm applied in Lam and Yan's method,

\subsection{The performance of the face recognition method}

In this section, we present a set of experiments using ORL face image database and IIS face image database (accessible at http://smart.iis.sinina.edu.tw) to illustrate and compare the performance of the proposed method and Lam and Yan's method for face recognition under varying poses. The comparisons of face recognition results at different seesaw rotation angles between the two methods are emphasized here.

ORL face image database includes 10 different face images of 40 distinct candidates (see examples in Fig. 10). Generally, faces in the images of this database satisfy the assumptions of our method: (1) $\theta_{x}$ is about in range of $-25^{\circ}$ to $25^{\circ}$, (2) $\theta_{y}$ is about in range of $-30^{\circ}$ to $30^{\circ}$, and (3) the two outside eye corners are not occluded. Lam and Yan [6] also conducted experiments based on ORL face image database. In their experiments, a face image in a nearly frontal view for each of the 40 candidates is chosen to construct the gallery and other 160 images, in which one half is in a nearly frontal view and the other half shows different amounts of pose variations, are selected to be test images. In these test images, there are only about 28 images with distinct seesaw rotation (with matched potential seesaw rotation angle $\theta_{x m-t}>5^{\circ}$ ). In our experiments, we utilized the same gallery named gallery A and used the same test images as test set 1 . Also, we formed test set 2 by 82 face images with distinct seesaw rotation. In IIS database, there are 100 persons and 30 pictures with different poses for each person (see examples in Fig. 14). We chose a nearly frontal view face image for each person from IIS database to construct another gallery $\mathrm{B}$. Accordingly, 800 images which meet assumptions (1), (2) and (3) of our method were chosen from IIS database to be test images called test set 3 . We automatically detect facial feature points and manually marked them on each image in gallery A, gallery $B$, test set 1 , test set 2 and test set 3 . In our system, the facial feature points are detected automatically. However for comparison of the recognition performance, the manually marked facial feature points were also used in the following experiments in the same way as that the automatically detected

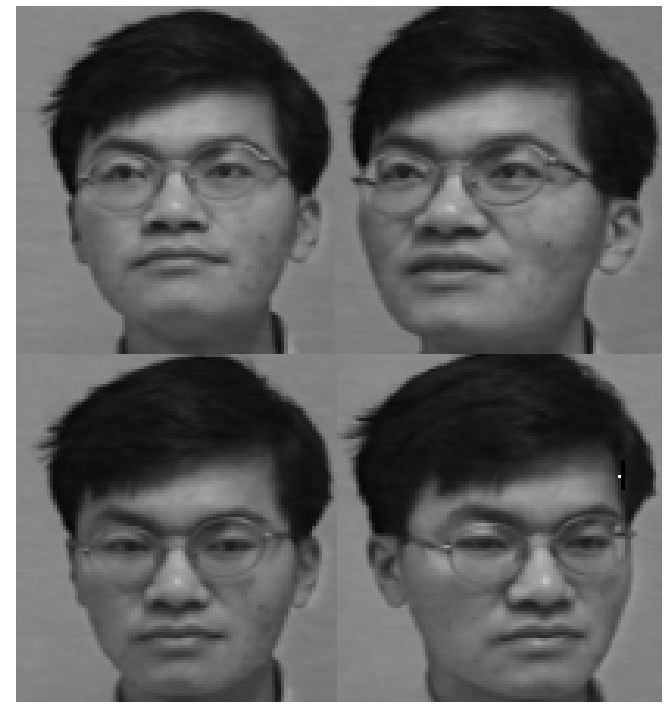

Fig. 14. Examples of face images in IIS face image database. 
ones were used. Thus there are 6 test data source TS1A, TS1M, TS2A, TS2M, TS3A and TS3M in our experiments. TS1A and TS1M represent the data sources use face images in Test set 1 with automaticlly detected and manually marked facial feature points respectively. The others are similar.

Overall recognition index $R=1-\left(1 / N_{t}\right) \sum_{i=1}^{N_{t}}(r(i) /(M-1))$ is used to evaluate the performance of the recognition methods in our experiments, where $\mathrm{M}$ is the number of faces in the gallery database, $N_{t}$ is the number of test images and $r(i)=0, \ldots, M-1$ is the position of the correct object on the similar list in which all objects in the gallery are sequenced according to their similarity to the test face. The gallery face that best matched the test one is placed on top of the list, while the least matched one is at the bottom. Thus $\mathrm{R}$ is in range of 0 to1. The larger $R$ is, the better result is. Actually it is an effective and convincing measurement of the recognition result. We also used recognition rate $R^{\prime}=N_{f} / N_{t}$, where $N_{f}$ is the number of test images that are recognized correctly, to measure the recognition performance in our experiments. In order to investigate the effect of the seesaw rotation to our face recognition method, we performed three experiments on TS2A, TS2M, TS3A, and TS3M. In the first and the second experiments, the performance of the geometric feature matching and facial feature template matching in our method are evaluated. The final face recogniton performance of our mehtod is investiged in the third experiment. For comparesion, the final recognition performance of Lam and Yan's method and the performance of both geometric feature matching and facial feature template matching in that mehtod were also be tested. The overall recognition index $R$ at different values of $\theta_{x m-t}$ (the matched potential seesaw rotation angle) of these experiments were illustrated in Fig. 15, (a)(b)(c) for test set 2, (d)(e)(f) for test set 3, (a)(d) for geometric feature matching, (b)(e) for facial feature template matching and (c)(f) for final face recogniton.

The experimental results shown in Figs. 15 indicate that the proposed method can achieve rubust face recognition even when the test faces hold distinct seesaw rotation and it has better recognition performance than Lam and Yan's method. It can be found, gernarally the larger seesaw rotation the test faces hold, the more obvious advantage of our mehtod is demonstrate compared to Lam and Yan's method. This advantage is obtained mainly because our method estimates the seesaw rotation and to some extent removes such pose variation on the test face classification

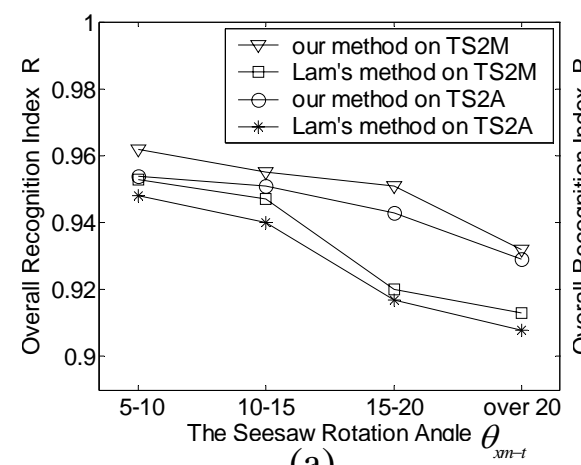

(a)

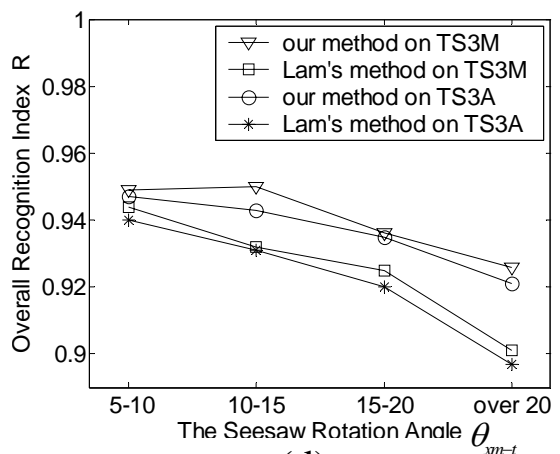

(d)

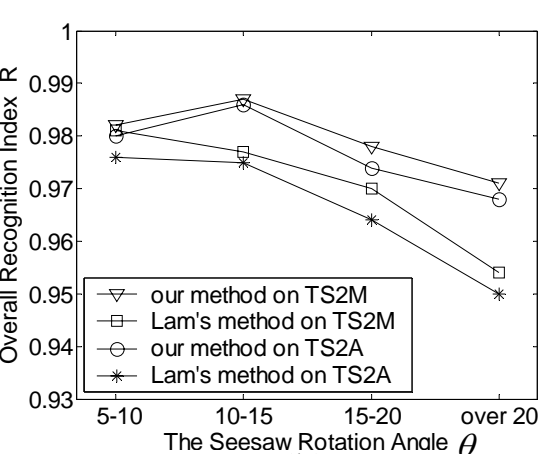

(b)

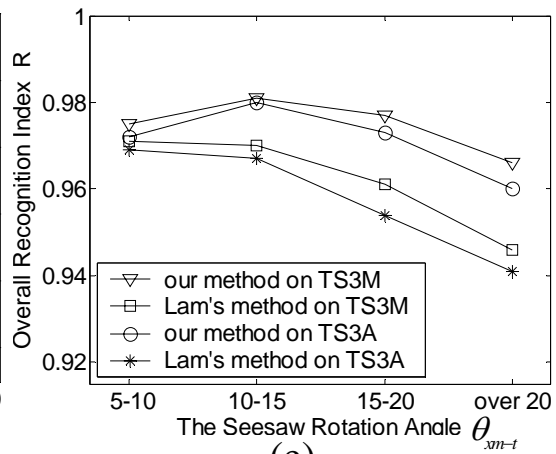

(e)

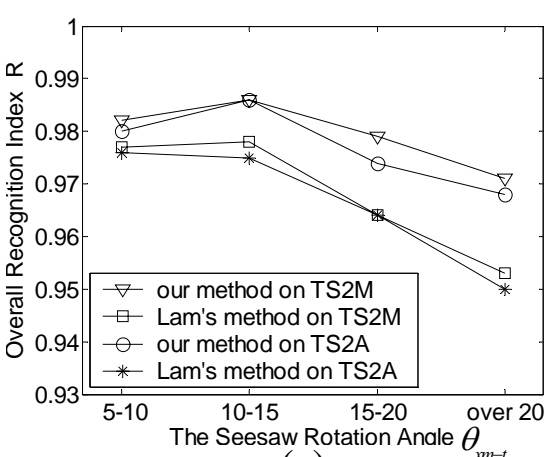

(c)

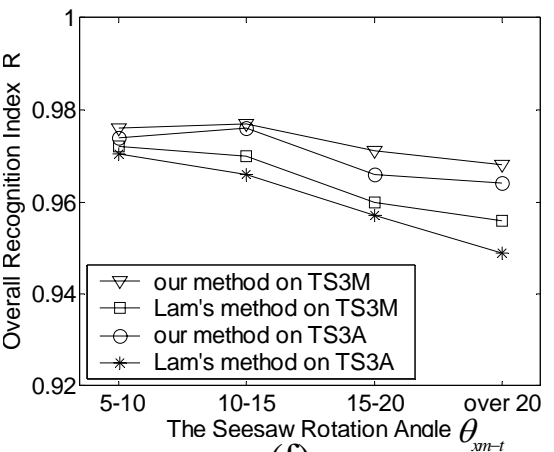

(f)

Fig. 15. The tested recognition performances at different seesaw rotation: (a)(b)(c) on test set 2, (d)(e)(f) on test set 3, (a)(d) for geometric feature matching, (b)(e) for template matching, (c)(f) for final face recognition. 
Table. 1 gives a comparison of overall recognition rate $R^{\prime}$ for our method and Lam and Yan's method on test set 1 , test set 2 and test set 3 (using manually marked facial points and detected facial feature points respectively). As there are only a few faces in test set 1 holding distinct seesaw rotations, the advantage of our method is not prominent. However since all faces have distinct seesaw rotation, the advantage of our method is more obvious in test set 2 and test set 3 .

\begin{tabular}{|c|c|c|c|c|c|c|}
\hline \hline & TS1A & TS1M & TS2A & TS2M & TS3A & TS3M \\
\hline \hline Our method & $87 \%$ & $91 \%$ & $78 \%$ & $81 \%$ & $77 \%$ & $80 \%$ \\
\hline am\&Yan's method & $84 \%$ & $89 \%$ & $67 \%$ & $69 \%$ & $70 \%$ & $74 \%$ \\
\hline
\end{tabular}

Table 1. Recognition rate comparison

\section{Conclusions}

This paper proposes a 3D model based pose invariant face recognition method that can recognize a face from its single nearly frontal view. The proposed method, using an analytic-to-holistic approach and a novel algorithm for estimation of ear points, can recognize faces of large rotation. Experiments were carried out to test the proposed ear point detection algorithm which was shown to have good accuracy. Using this algorithm and an improved face model, we develop a more general pose estimation algorithm. Experimental results show that the proposed pose estimation algorithm can effectively estimate both the seesaw rotation and the sidespin rotation of the test face. Based on the general pose estimation, pose invariant features and so more accurate face recognition can be obtained by corresponding template matching and corresponding geometric feature matching. Experimental results show that the proposed method is more robust to pose variation compared to Lam and Yan's method and has a good performance for pose invariant face recognition.

\section{References}

[1] R. Chellappa, C. Willson, and S. Sirohey, "Human and Machine Recognition of Faces: A Survey," Proc. IEEE, vol.83 (5), pp. 705-740, 1995.

[2] M. A. Grudin, “On internal representations in face recognition systems,” Pattern Recognition, vol. 33 (7), pp. 1161-1177, 2000.

[3] A. Pentland, "Looking at People: Sensing for Ubiquitous and Wearable Computing," IEEE Trans. Pattern Analysis and Machine Intelligence, vol. 22 (1), pp. 107-119, 2000.

[4] F. Mokhtarian and R. Suomela, "Robust Image corner Detection Through Curvature Scale Space," IEEE Trans. on Pattern Analysis and Machine Intelligence, vol. 20 (12), pp. 1376-1381, 1998.

[5] A. Rosenfeld and E. Johnston, “Angle detection in digital curves,” IEEE Trans. Comput., vol. C-22, pp.875-878, 1973.

[6] K. M. Lam and H. Yan, “An Analytic-to-Holistic Approach for Face Recognition Based on a Single Frontal View,” IEEE Trans. Pattern Analysis and Machine Intelligence, vol. 20 (7), pp. 673-686, 1998.

[7] R. Brunelli and T. Poggio, "Face Recognition: Features vs. Templates,” IEEE Trans. Pattern Analysis and Machine Intelligence, vol. 15 (10), pp. 1042-1053, Oct. 1993.

[8] P. N. Belhumeur, J.P. Hespanha, and D.J. Kriegman, "Eigenfaces vs. Fisherfaces: Recognition Using Class Specific Linear Projection,” IEEE Trans. Pattern Analysis and Machine Intelligence, vol. 19 (7), pp.711-720, 1997.

[9] S. Li and J. Lu, "Face Recognition Using Nearest Feature Line,” IEEE Trans. Neural Net- works, vol. 10 (2), pp. 439-443, 1999. 
[10] M. S. Bartlett, J.R. Movellan, and T.J. Sejnowski, "Face Recognition by Independent Component Analysis,” IEEE Trans. Neural Networks, vol. 13 (6), pp. 1450 -1464, 2002.

[11] J.-T. Chien and C.-C. Wu, "Discriminant Waveletfaces and Nearest Feature Classifiers for Face Recognition,” IEEE Trans. Pattern Analysis and Machine Intelligence, vol. 24 (12), pp. 1644-1649, 2002.

[12] J. W. Lu, K.N. Plataniotis, and A. N. Venetsanopoulos, "Face Recognition Using Kernel Direct Discriminant Analysis Algorithms," IEEE Trans. Neural Networks, vol. 14 (1), pp. 117-126, 2003.

[13] Y. S. Gao and M.K.H. Leung, "Face Recognition Using Line Edge Map,” IEEE Trans. Pattern Analysis and Machine Intelligence, vol. 24 (6), pp. 764 -779, 2002.

[14] A. M. Martinez, "Recognition of Partially Occluded and/or Imprecisely Localized Faces Using a Probabilistic Approach," in Proc. IEEE Conference on Computer Vision and Pattern Recognition, vol. (1), pp. 712-717, 2000.

[15] L. Wiskott, J. M. Fellous, N. Kruger, and C. von der Malsburg, "Face Recognition by Elastic Bunch Graph Matching,” IEEE Trans. Pattern Analysis and Machine Intelligence, vol. 19 (7), pp. 775-779, 1997.

[16] F. J. Huang, Z. Zhou, H. J. Zhang, and T. Chen, "Pose invariant face recognition," in Proc. of 4th IEEE International Conference on Automatic Face and Gesture Recognition, pp.245-250, March 2000.

[17] Y. Gao, M. K. H. Leung, W. Wang and S. C. Hui, "Fast Face Identification under Varying Pose from a Single 2-D Model View,” IEE Proceedings, vol. 148 (4), pp. 248 -253, 2001.

[18] Murase, and S.K. Nayar., "Learning and Recognition of 3D Objects from Appearance" in IEEE 2nd Qualitative Vision Workshop, New York, NY, June 1993.

[19] E. Demir, L. Akarun, and E. Alpaydin, “Two-stage Approach for Pose Invariant Face Recognition,” in Proc. International Conference on Acoustics, Speech, and Signal Processing, vol.6, pp. 5-9, Jun. 2002.

[20] O. De Vel and S. Aeberhard, “Line-based Face Recognition under Varying Pose,” IEEE Trans. Pattern Analysis and Machine Intelligence, vol. 21 (10), pp. 1081 -1088, 1999.

[21] G. C. Feng, and P.C. Yuen, "Recognition of Head-and-Shoulder Face Image Using Virtual FrontalView Image,” IEEE Trans. Systems, Man and Cybernetics, Part A, vol. 30 (6), pp. 871 -882, 2000.

[22] Q. Chen, H. Y. Wu, S. Shioyama, and T. Shimada, "Head Pose Estimation Using Both Color and Feature Information,” in Proc. 15th International Conference on Pattern Recognition, vol. 2, pp. 842 845, Sept. 2000.

[23] V. Blanz, S. Romdhani, and T. Vetter, "Face Identification Across Different Poses and Illuminations with a 3D Morphable Model," in Proc. IEEE 5th International Conference on Automatic Face and Gesture Recognition, pp. 100-105, 20-21 May 2002.

[24] V. Blanz and T. Vetter, "Face Recognition Based on Fitting a 3D Morphable Model,” IEEE Trans. Pattern Analysis and Machine Intelligence, vol. 25 (19), pp. 1063 -1074, 2003. 